\title{
Sugar, fat, saturated and trans fatty acid contents in Iranian cereal-based baked products
}

\author{
Zahra HADIAN ${ }^{1 *}$, Amin MOUSAVI KHANEGHAH² (D)
}

\begin{abstract}
The intake levels of sugar and fats, including unsaturated fatty acids (USFs), trans-fatty acids (TFAs), and saturated fatty acids (SFAs), should be monitored to prevent the prevalence of non-communicable diseases. Cereal-based bakery products are one of the significant sources of dietary fat and sugar intake throughout the world. This study evaluates the trend of fat and sugar in Iranian bakery products, focusing on saturated and TFAs and their implication for public health. In this regard, 132 industrial and traditional cereal-based-backed products were randomly collected from the Tehran market. The total fat, SFAs, TFAs, unsaturated fatty acids (UFAs), and sugar of each bakery product were determined. The fatty acid compositions of these products were evaluated according to the direct methylation method. Our results indicated that total fat in Iranian bakery products ranged from $8.99 \pm 2.95 \%$ (sponge cake) to $24.92 \pm 7.86 \%$ (puffed product). Total sugar varied from $11.12 \pm 1.89 \%$ (pirashki) to $30.38 \pm 13.11 \%$ (funnel pastry). Among fifteen different types of bakery products, the highest TFAs and UFAs levels were detected in simple biscuits $(0.52 \pm 0.50 \%)$ and $(17.20 \pm 8.71 \%)$, respectively. The present study indicated that bakery products' sugar and fat content as risk factors of non-communicable diseases was not higher than those of other countries.
\end{abstract}

Keywords: trans-fatty acid; saturated-fatty acid; bakery products; iran; non-communicable diseases.

Practical Application: This study is the first national report for health intervention plans and strategies programs due to the high consumption of commercial and traditional cereal-based-baked products.

\section{Introduction}

Sweet bakery products are among the world's most popular foods owing to their favorable sensory attributes and long shelf life (Pinto et al., 2019). Fats and oils such as shortening, margarine, butter, and vegetable oils have been incorporated blended or alone at various ratios in formulations of bakery products due to their favorable effects such as improving flavor and structure, aiding the absorption of fat-soluble vitamins, and supplying energy (37 kJ/g fat) (Hercegová et al., 2019; Omeroglu \& Ozdal, 2020). At the same time, these fats and oils are primary sources of trans-fatty acids (TFA) and saturated fatty acids (SFA) in bakery products. For instance, crackers, cake, and cookies account for $40 \%$ of TFA intake (O'Fallon et al., 2007). High intake of TFA can cause violations in high-density lipoproteins (HDL) and low-density lipoproteins (LDL) (Albuquerque et al., 2018; Priego-Capote et al., 2007; Richter et al., 2009; Stanner \& Spiro, 2020). Therefore, excessive TFA intake and SFA are correlated to a higher risk of diabetes, coronary heart disease (CHD), breast cancer, obesity, and sudden death (Alioğlu \& Özülkü, 2020; Belc et al., 2019; Mozaffarian et al., 2007; Trattner et al., 2015). Similarly, higher sugar intake is directly connected to increased body weight, a severe risk factor for cancer and diabetes (Pușcaș et al., 2020). To prevent these adverse effects, the World Health Organization (WHO) suggested that the daily intake of total fat, SFA, and TFA should not exceed $30 \%, 10 \%$, and $1 \%$ of total calories, respectively (Saghafi et al., 2018). Besides, WHO recommends that the daily intake of simple sugars should be reduced to $10 \%$ of total energy, and according to their reports, reducing sugar intake to less than $5 \%$ of total energy can lead to even more health-beneficial influences (World Health Organization, 2015). In the same vein, reducing TFA consumption and SFA consumption is another significant step suggested by WHO to be taken by different countries to minimize non-communicable diseases (NCDs) (Albuquerque et al., 2018; Arcand et al., 2014). The effect of reducing fat intake to less than $30 \%$ of the total daily energy on weight gain prevention among adults has been wellestablished (Albuquerque et al., 2018; Colón-Ramos et al., 2014; Hooper et al., 2012; Zuchowska-Grzywacz \& Kowalska, 2019). In addition to the overall fat intake, TFA and SFA intakes are influential in studying NCDs' risk factors (O'Sullivan, 2020; World Health Organization, 2003). In this regard, different strategies, including new formulation, labelling, enacting legislation, and education, have been adopted throughout the world that provide nutritional needs as well as health advantages (Albuquerque et al., 2018; ColónRamos et al., 2014; Dordevic et al., 2020; (Albuquerque et al., 2018; Colón-Ramos et al., 2014; Dordevic et al., 2020; FuentesArismendy et al., 2021; Lucas et al., 2020; Sarifudin et al., 2021).

Thus, it seems that analysis of fatty acid composition in bakery products is a needful issue to monitor these approaches' performance and raise public awareness concerning their daily intake estimation and health implications. As studies showed, sugar consumption in Iran is 30\% higher than that of the global standard. According to a recent study conducted by 
Dadkhah et al., $40 \%$ of total energy intake was provided by snacks in students of a primary school in Tehran (Amini et al., 2014). In another research, Asgary et al. (2009) found out that TFAs in Iranian fast foods were higher than those recommended by general diet guidelines (Asgary et al., 2009). Based on the literature, there was still a lack of research on fatty acid compositions of popular bakery products in Iran. Thus, the present study's objectives were to determine the sugar, total fat, and fatty acid composition of industrial and traditional-cereal-based products widely marketed in Iran.

\section{Materials and methods}

\subsection{Reagents and standards}

All reagents and solvents were of analytical grade. Methanol, sulfuric acid, $\mathrm{n}$-hexane, potassium hydroxide, and Fehling's solution was purchased from Merck (Darmstadt, Germany). The certified standard of fatty acid methyl ester (FAME) mixture was achieved from Supelco (Bellefonte, PA., U.S.A.) and nonadecanoic acid (C19:0) as internal standard was provided by Sigma Aldrich Co. (St. Louis, Missouri, U.S.A.).

\subsection{Sample description}

This study is cross-sectional research on food and nutrition policy implications for monitoring nutritional risk factors in Iran IR. One hundred thirty-two samples comprising fifteen different brands of industrial bakery products group including cake, sponge cake, cracker, koluche, simple biscuit, non-simple biscuit, pirashki, puffed products, and wafer, and traditional bakery products such as cake, fermented pastry (e.g., Danmarki and Napeloni), funnel pastry (e.g., Bamiyeh and Keshmeshi), Koluche, layered pastry (e.g., Baghlava), non-fermented pastry (e.g., Mikado and Zaban) and Pirashki, were purchased from local supermarkets and retail-markets in Tehran, Iran, during April 2016 to June 2016. The study protocol was conducted by the National Nutrition and Food Technology Research Institute. Each of the samples was coded with a three-digit number, stored in $4{ }^{\circ} \mathrm{C}$, and analyzed before expiry dates. Collected samples included the significant manufacturers of bakery products in Iran.

\subsection{Moisture, total fat, and sugar contents determination}

The moisture content of traditional and industrial cerealbased-baked bakery samples was measured by vacuum oven (Memmert V09, Schwabach, Germany) at $100-130{ }^{\circ} \mathrm{C}$ temperature according to the International organization for Standardization by brief modification (International Organization for Standardization, 2009).

Total lipid extraction was performed using the method described by Fölch et al. (Folch et al., 1957). The sugar content of samples was evaluated according to the official method developed and designated by the Iranian National Standards Organization (Institute of Standards and Industrial Research of Iran, 2016). All tests were performed in triplicates.

\subsection{Preparation of methyl esters of fatty acids}

The fatty acid profile of bakery products was determined by the direct methylation method described by O'Fallon et al. (2007) (O'Fallon et al., 2007) and ISO 12966-1 (International Organization for Standardization, 2014). First, samples were ground in a coffee bean grinder at room temperature. A level of $700 \mathrm{mg}$ of wet samples was located in a $16 \times 125 \mathrm{~mm}$ screwcap Pyrex culture tube, to which $0.6 \mathrm{ml}$ of $\mathrm{MeOH}$ containing internal standard ( $0.3 \mathrm{mg}$ of $\mathrm{C} 19: 0 \mathrm{~mL}^{-1}$ of $\mathrm{MeOH}$ ) was included. After that, $0.7 \mathrm{ml}$ of $10 \mathrm{~N} \mathrm{KoH}$ was added to the Pyrex tube containing the samples. The tubes were incubated for $1.5 \mathrm{~h}$ in a water bath at $55^{\circ} \mathrm{C}$ with occasional shaking ( $5 \mathrm{~s}$ every $20 \mathrm{~min}$ ) using an I.K.A. Vortex Genius 3 (IKA-Werke GmbH \& CO.KG, Germany). After using a cold water bath to cool the samples down, the samples were added to each sample with $0.58 \mathrm{ml}$ of $\mathrm{H}_{2} \mathrm{SO}_{4}(24 \mathrm{~N})$ in water. The tubes were inverted to ensure proper mixing, and after the addition of $\mathrm{K}_{2} \mathrm{SO}_{4}$ solution, the tubes were incubated in a water bath at $55^{\circ} \mathrm{C}$ for $1.5 \mathrm{~h}$ with occasional shaking. After FAME synthesis, the tubes were cooled down with a cold water bath, and $2 \mathrm{~mL}$ of hexane was added and mixed for $5 \mathrm{~min}$. Subsequently, the tubes were centrifuged for $10 \mathrm{~min}$ at $4500 \times \mathrm{g}$, and the upper hexane layer containing the FAME was placed into the GC vial. Finally, the GC vials were placed at $-20{ }^{\circ} \mathrm{C}$ until GC analysis. Then, FAs concentrations were determined by GC-FID (Varian CP3800, U.S.A.) equipped with an HP-88 column $(100 \mathrm{~m} \times 0.25 \mathrm{~mm}$ ID, $0.2 \mu \mathrm{m})$. The operating conditions were set as follows: the initial temperature was set at $50{ }^{\circ} \mathrm{C}$ and gradually increased $\left(5^{\circ} \mathrm{C}\right.$, every $\left.5 \mathrm{~min}\right)$ up to $160^{\circ} \mathrm{C}$, and then reached to $180^{\circ} \mathrm{C}$ with a ramp of $20^{\circ} \mathrm{C} \mathrm{min}{ }^{-1}$ and finally increased to $220^{\circ} \mathrm{C}$. The temperature of the injector and detector were $250^{\circ} \mathrm{C}$ and $260^{\circ} \mathrm{C}$, respectively. The flow rate of helium as the carrier gas was $2 \mathrm{ml} / \mathrm{min}$, and the injection volume was $0.2 \mu \mathrm{m}$. All FAs were reported as the percentage of total FAs (Mossoba et al., 2003). The contents of sugar, TFAs, SFAs, UFAs, and total lipids are presented in $100 \mathrm{~g}$ food. The TFA content is further presented per $100 \mathrm{~g}$ total lipids. Total fat indicated the sum of triglycerides, phospholipids, wax ester, and sterols. SFAs attributed to the sum of 14 isomers (C8:0, C10:0, C12:0, C14:0, C15:0, C16:0, C17:0, C18:0, C19:0, C20:0, C10:0, C22:0, C23:0 and C24:0), and TFA indicated the sum of 8 isomers (C18:1t, $\mathrm{C} 18: 2 \mathrm{t}$ and $\mathrm{C} 18: 3 \mathrm{t})$. USFs referred to the sum of 11 isomers (C14:1; C16:1; C17:1; C18:1cisn6; C18:1cisn9; C18:2cis9, 12; C18:3 cis9, 12, 15; C20:1; C20:2; C20:3n6 and C22:1). Figure 1 shows a typical chromatogram of a sample analyzed.

\subsection{Statistical analysis}

Descriptive analyses were performed by using SPSS software V. 26 (I.B.M. Analytics, U.S.A.). The data (mean \pm SD) indicated triplicate determination and analyzed by Mann-Whitney test, one-sample $\mathrm{t}$-test, and independent $\mathrm{t}$-test to compare fat and sugar contents in samples and compare the results of fat and sugar content with those target levels permitted by the Iranian National Standards Organization. Kruskal-Wallis test was used to compare fat and sugar levels in each group. The significant differences among the mean values were calculated at the level of P-value $\leq 0.05$. 


\section{Results and discussion}

\subsection{Trans-fatty acids and saturated fatty acids profiles}

The trans-fatty acids (TFAs) contents of industrial and traditional bakery products are shown in Table 1. In the industrial category, the maximum level of TFAs was found in simple biscuits $(0.52 \pm 0.50 \%)$ and then in cake $(0.41 \pm 0.55 \%)$. The minimum level was found in the puffed product $(0.17 \pm 0.03 \%)$. The highest TFAs level in the traditional category was determined in funnel pastry $(0.48 \pm 0.69 \%)$ and followed by fermented pastry $(0.35 \pm 0.41 \%)$. Total trans fatty acids in bakery products (132 samples) were ranged from $0.01 \%$ to $2.88 \%$. The content of $\mathrm{C} 18: 3 \mathrm{t}$ in the industrial group was significant $(\mathrm{P}=0.001)$. Among the TFAs content in industrial and traditional samples, the notable content was related to $\mathrm{C} 18: 1 \mathrm{t}$ that belonged to cake $(2.80 \%)$ and funnel pastry (1.84\%), respectively. As a result, indicate the content of

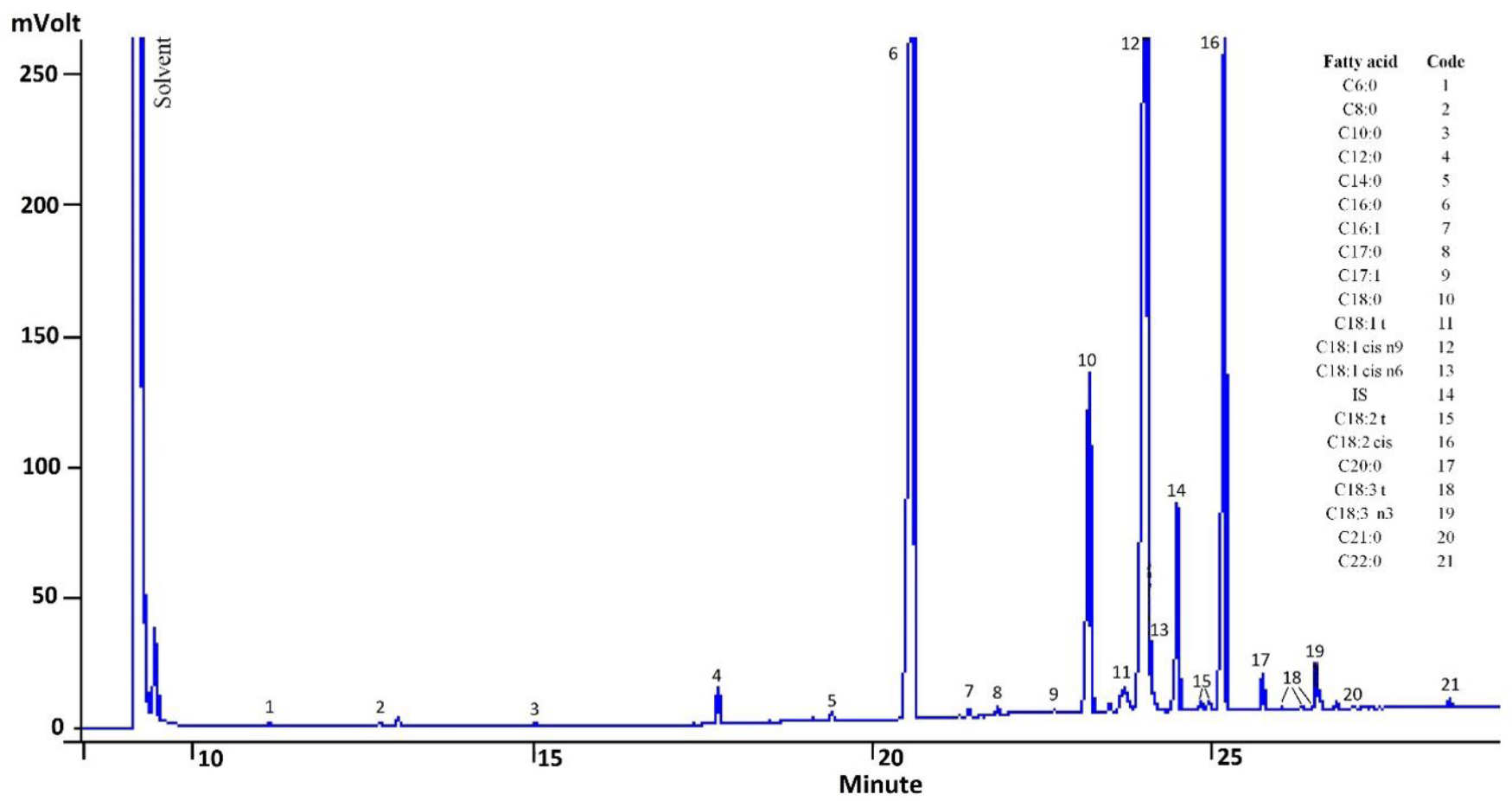

Figure 1. Gas chromatogram of fatty acids profile of bakery sample

Table 1. Trans fatty acids (g/100 g food) of Iranian bakery products.

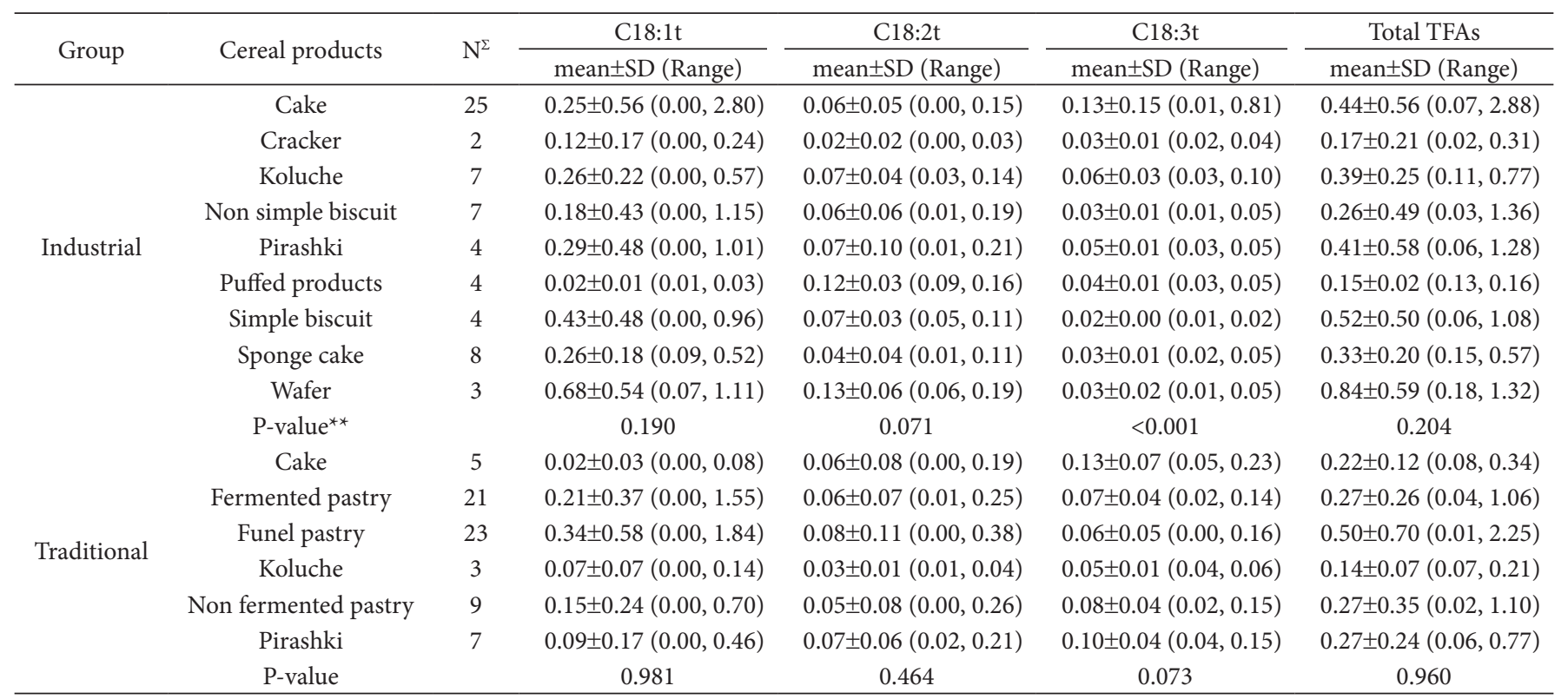

$\Sigma$. Number of samples; ${ }^{* *}$ The Kruskal Wallis test. 
total trans fatty acids in industrial and traditional samples was not significant $(P \geq 0.05)$.

Also, according to Table 1, there were no zero trans in Iranian bakery products. Total TFAs ranged from $0.14 \pm 0.07 \%$ to $0.52 \pm 0.5 \%$, and maximum and minimum levels were determined in simple biscuit and koluche, respectively. Partially hydrogenated oils in Iranian food basket consumption have particular importance, with a mean per capita intake of $14 \mathrm{~g} / 1000 \mathrm{kcal}$. Several studies demonstrated that trans-fat causes heart attack and death. In most countries, the critical strategy was implemented through different approaches, including legislative action (limited to $2 \%$ of total fat content in foods) or by banning the consumption of partially hydrogenated oils (Mozaffarian et al., 2007).

The usage of partially hydrogenated fats, including bakery fats, margarine, and shortenings in the formulation of bakery products, increases SFAs and TFAs in these products (Karabulut, 2007). Modified hydrogenation and reducing hydrogenated fats in the formulation of bakery products can lead to less saturated and trans fatty acid contents (Menaa et al., 2013). In this view, Vicario et al. (2003) studied and characterized the fatty acid profile of bakery products in Spain and reported a range of $0.15-15.94 \% \mathrm{w} / \mathrm{w}$ trans fatty acids/total fatty acids in these products (Vicario et al., 2003). In another study, total TFA in the Portuguese food market was analyzed, and the mean value was reported as $0.47 \mathrm{~g} / 100 \mathrm{~g}$ food ranging $0.01-6.02 \%$ in which biscuits, wafers, and cookies had the highest TFA content (Costa et al., 2016). Chung et al. (2013) evaluated trans fatty acids in the Hong Kong Food Supply and reported that the mean value of TFA for bakery products ranged from $0.09(\mathrm{~g} / 100 \mathrm{~g}$ food) in sponge cakes to $0.9 \mathrm{~g}$ in doughnuts and, SFAs varied from 2.4 ( $\mathrm{g} / 100 \mathrm{~g}$ food) in the Chinese fried dough to $14 \mathrm{~g}$ in creamy biscuits (Chung et al., 2013). The total SFA was higher than $40 \mathrm{~g} / 100 \mathrm{~g}$ fatty acid in Turkish bakery products, and total TFA varied from 0.99 in petit-beurre biscuits to $17.71(\mathrm{~g} / 100 \mathrm{~g}$ fatty acids) in traditional wheat flour cookies (Karabulut, 2007). Thus, the TFA content of bakery products in Iran was lower than those in Spain, Portugal, and Turkey; nevertheless, it was almost similar to the TFA of bakery products in Hong Kong. In addition, SFAs content of bakery products in Iran was close to those in Hong Kong and Turkey. It is now well established that the risk of cardiovascular disease, diabetes, systematic inflammation, and sudden death is correlated with high consumption of trans fatty acids (Saadeh et al., 2015).

On the other hand, a high intake of SFAs is also correlated to a higher risk of cancer (Chajès et al., 2008; Slattery et al., 2001). The FAO/WHO recommends a maximum of $1 \%$ of the energy received from trans fatty acids (Nishida et al., 2004). In this study, the most common TFA was elaidic acid (C18:1 t-9) among measured trans fatty acids. Similarly, Nazari et al. (2012) reported elaidic acid as the prominent TFA found in Iranian bakery and snack food products (Nazari et al., 2012).

Results revealed that the SFAs in Iranian bakery products ranged from $2.17 \pm 3.06 \%$ to $12.45 \pm 3.19 \%$ (Table 2). The highest level of saturated fatty acids among the fifteen types evaluated products was found in puffed products (12.45 $\pm 3.19 \%)$. The maximum SFAs levels in traditional bakery samples were obtained in koluche $(7.79 \pm 2.46 \%)$ and followed by fermented pastry $(7.78 \pm 3.72 \%)$.
The minimum level $(3.80 \pm 0.25 \%)$ was found in the cake. SFAs contents of the industrial products ranged from $2.17 \pm 3.06 \%$ (cracker) to $12.45 \pm 3.19 \%$ (puffed product). As Table 2 shows, the major saturated fatty acids in analyzed samples were lauric acid (C12:0), myristic acid (C14:0) and palmitic acid (C16:0), and stearic acid (C18:0), while a very small amount of other SFAs was detected. The mean of SFAs content in total industrial bakery samples was between $2.17 \%$ and $12.45 \%$, and in total traditional bakery, samples were between $3.80 \%$ and $7.79 \%$, respectively. The content of SFAs in the industrial group was significant $(\mathrm{P}=0.003)$; similar results were found for the traditional group $(\mathrm{P}=0.036)$.

\subsection{The unsaturated fatty acid profile}

Unsaturated fatty acids (UFAs) contents of different bakery samples are presented in Table 3. In the industrial category, the maximum and minimum level of UFAs was found in the cracker (17.20 $\pm 8.71 \%)$ and sponge cake $(4.41 \pm 0.84 \%)$, respectively. Besides traditional bakery products, the highest and lowest UFAs levels were observed in non-fermented pastry $(14.60 \pm 4.29 \%)$ and koluche $(7.26 \pm 1.92 \%)$, respectively. Among the identified USFAs in both groups, the contents of C18:1cisn9 and C18:2cis were higher than other fatty acids. Overall, comparing USFAs content in each group revealed a statistically significant difference $(\mathrm{P} \leq 0.05)$.

As shown in Table 3, UFAs (cis) content in bakery products ranged from $4.41 \pm 0.84 \%$ to $17.20 \pm 8.7 \%$ in sponge cake and cracker, respectively. Oleic acid (C18:1) and linoleic acid (C18:2) levels were considerably higher in all bakery products compared to other measured UFSs. Similarly, linoleic acid as an essential FA was found in high levels in European bakery products (Cercaci et al., 2006).

\subsection{Total fat and sugar contents}

Total fat and sugar in industrial and traditional basedcereal products are shown in Table 4. Among industrial bakery products, the maximum level of total fat content was observed in the puffed product $(24.92 \pm 7.86 \%)$ followed by the cracker (19.59 $\pm 5.77 \%)$. Likewise, the minimum level was found in sponge cake $(8.99 \pm 2.95 \%)$. In the traditional category, non-fermented pastry $(22.24 \pm 7.02 \%)$ and koluche $(15.30 \pm 3.61 \%)$ had to contain the maximum and minimum total fat levels, respectively.

The maximum sugar levels of industrial bakery products were observed in sponge cake $(29.92 \pm 6.02 \%)$ followed by koluche $(27.31 \pm 5.07 \%)$. As the same way, the minimum level of sugar was found in pirashki (17.17 $\pm 9.38 \%)$. The sugar levels in traditional bakery samples were in ranged from $11.12 \pm 1.89 \%$ (pirashki) to $30.38 \pm 13.11 \%$ (funnel pastry).

Generally, all samples had to contain TFAs, but lower than the target limit set by the Iranian National Standards Organization (2\%). Among fifteen different bakery products, the highest and lowest FAs levels were observed in puffed products and sponge cake, respectively. Moreover, the highest and lowest sugar contents among bakery products were found in funnel pastry and traditional pirashki, respectively. In the present study, the fat content of bakery products ranged from $8.99 \pm 2.95 \%$ to $24.92 \pm 7.86 \%$. As results in Table 4 indicated, in comparing 


\begin{tabular}{|c|c|c|c|c|c|c|c|c|c|c|}
\hline 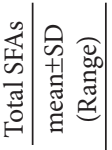 & 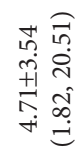 & 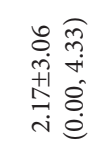 & 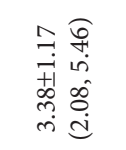 & 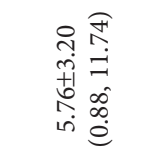 & 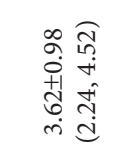 & 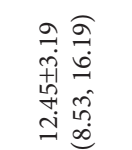 & 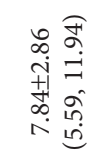 & 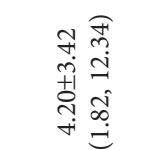 & 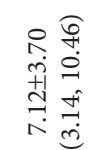 & ڤ̊ \\
\hline 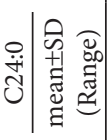 & 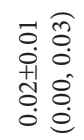 & $\begin{array}{l}8 \\
8 \\
0 \\
0 \\
0 \\
11 \\
0 \\
0 \\
0 \\
0\end{array}$ & $\begin{array}{l}8 \\
0 \\
0 \\
0 \\
0 \\
0 \\
0 \\
0 \\
0 \\
0\end{array}$ & 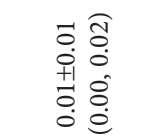 & 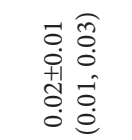 & $\begin{array}{ll}8 & \widehat{o} \\
0 & 0 \\
0 & 0 \\
11 & \hat{\delta} \\
0 & 0 \\
0 & \stackrel{0}{0}\end{array}$ & $\begin{array}{ll} & \\
0 & \widehat{\Xi} \\
0 & 0 \\
0 & 0 \\
0 & 0 \\
0 & 0 \\
0 & 0\end{array}$ & $\begin{array}{ll}8 & \widehat{0} \\
0 & 0 \\
+1 & 0 \\
0 & 0 \\
0 & 0 \\
0 & 0\end{array}$ & $\begin{array}{l}8 \\
0 \\
0 \\
0 \\
+1 \\
0 \\
0 \\
0 \\
0\end{array}$ & $\overrightarrow{0}$ \\
\hline 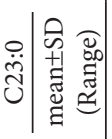 & 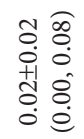 & 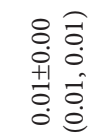 & 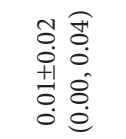 & $\begin{array}{ll}\overrightarrow{0} & \widehat{0} \\
0 & 0 \\
0 & 0 \\
+1 & 0 \\
0 & 0 \\
0 & 0\end{array}$ & 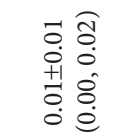 & $\begin{array}{l}\overrightarrow{0} \\
0 \hat{0} \\
0 \\
+1 \\
0 \\
0 \\
0 \\
0\end{array}$ & 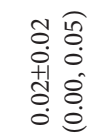 & 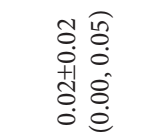 & $\begin{array}{l}8 \\
0 \\
0 \\
+0 \\
+1 \\
0 \\
0 \\
0\end{array}$ & $\stackrel{\infty}{\stackrel{\infty}{\hat{b}}}$ \\
\hline 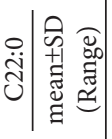 & 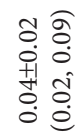 & 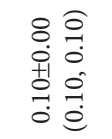 & 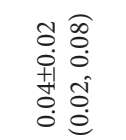 & 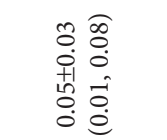 & 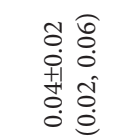 & $\begin{array}{ll} & 0 \\
0 & \widehat{L} \\
0 & 0 \\
0 & 0 \\
0 & 0 \\
0 & 0 \\
0 & 0 \\
0\end{array}$ & $\begin{array}{ll}0 & \widehat{O} \\
0 & 0 \\
0 & 0 \\
+1 & 0 \\
0 & 0 \\
0 & 0 \\
0 & 0\end{array}$ & $\begin{array}{ll} & \widehat{O} \\
0 & 0 \\
0 & 0 \\
+1 & 0 \\
0 & 0 \\
0 & 0\end{array}$ & 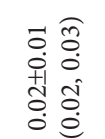 & $\begin{array}{l}\vec{\Delta} \\
\dot{0} \\
\dot{v}\end{array}$ \\
\hline 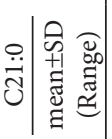 & 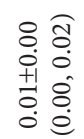 & 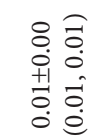 & $\begin{array}{l}8 \\
0 \\
0 \\
0 \\
0 \\
0 \\
0 \\
0 \\
0\end{array}$ & 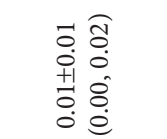 & $\begin{array}{l}\overrightarrow{0} \widehat{o} \\
0 \\
0 \\
0 \\
0 \\
0 \\
0 \\
0\end{array}$ & $\begin{array}{l}8 \\
0 \\
0 \\
0 \\
01 \\
0 \\
0 \\
0 \\
0\end{array}$ & $\begin{array}{l}8 \\
8 \\
0 \\
0 \\
0 \\
0 \\
0 \\
0 \\
0\end{array}$ & $\begin{array}{l}8 \\
8 \\
0 \\
0 \\
0 \\
0 \\
0 \\
0 \\
0\end{array}$ & $\begin{array}{l}\overrightarrow{0} \\
0 \\
0 \\
0 \\
0 \\
0 \\
0 \\
0\end{array}$ & 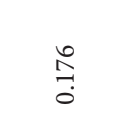 \\
\hline 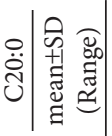 & 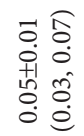 & 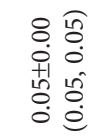 & $\begin{array}{l}\overrightarrow{0} \\
0 \\
0 \\
0 \\
0 \\
0 \\
0 \\
0 \\
0\end{array}$ & $\begin{array}{ll} & 0 \\
0 & 0 \\
0 & 0 \\
+1 & 0 \\
0 & 0 \\
0 & 0 \\
0 & 0\end{array}$ & $\begin{array}{ll} & 0 \\
0 & 0 \\
0 & 0 \\
11 & 0 \\
0 & 0 \\
0 & 0 \\
0 & 0\end{array}$ & 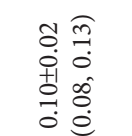 & 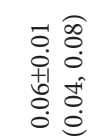 & 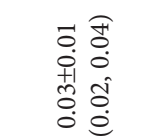 & $\begin{array}{l}m \\
0 \\
0 \\
0 \\
01 \\
0 \\
0 \\
0 \\
0\end{array}$ & $\begin{array}{l}\vec{\Xi} \\
\dot{0}\end{array}$ \\
\hline 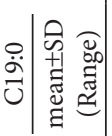 & 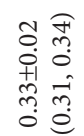 & $\begin{array}{l}8 \\
0 \\
0 \\
0 \\
0 \\
0 \\
0 \\
0 \\
0\end{array}$ & $\begin{array}{l}8 \\
8 \\
0 \\
0 \\
0 \\
0 \\
0 \\
0 \\
0\end{array}$ & $\begin{array}{l}8 \\
8 \\
0 \\
0 \\
0 \\
0 \\
0 \\
0 \\
0 \\
0\end{array}$ & $\begin{array}{l}8 \\
8 \\
0 \\
0 \\
0 \\
0 \\
0 \\
0 \\
0\end{array}$ & 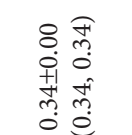 & 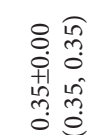 & $\begin{array}{l}8 \\
\dot{0} \\
0 \\
0 \\
01 \\
0 \\
0 \\
0\end{array}$ & $\begin{array}{l}8 \\
0 \\
0 \\
0 \\
0 \\
0 \\
0 \\
0 \\
0\end{array}$ & $\begin{array}{l}\text { ồ } \\
\text { 苟 }\end{array}$ \\
\hline 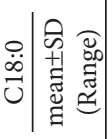 & 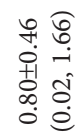 & 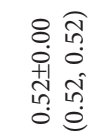 & 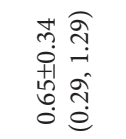 & \begin{tabular}{ll} 
& \multicolumn{1}{c}{} \\
0 & 0 \\
0 & 0 \\
01 & 0 \\
0 & 0 \\
0 & 0 \\
0 & 0
\end{tabular} & 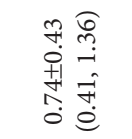 & 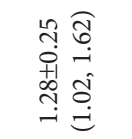 & 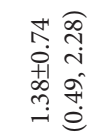 & 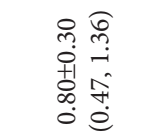 & 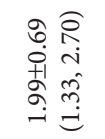 & $\begin{array}{l}\infty \\
\stackrel{0}{0} \\
0\end{array}$ \\
\hline 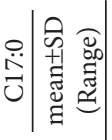 & 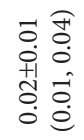 & $\begin{array}{l}8 \hat{0} \\
0 \\
0 \\
+1 \\
0 \\
0 \\
0 \\
0\end{array}$ & 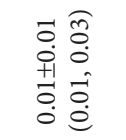 & $\begin{array}{ll}n & \widehat{n} \\
0 & 0 \\
0 & 0 \\
+1 \\
0 & 0 \\
0 & 0 \\
0 & 0\end{array}$ & $\begin{array}{l}0 \widehat{0} \\
0 \\
0 \\
+0 \\
+1 \\
0 \\
0 \\
0\end{array}$ & 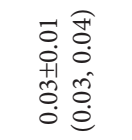 & \begin{tabular}{ll} 
& \multicolumn{1}{c}{} \\
0 & 0 \\
0 & 0 \\
+1 & 0 \\
0 & 0 \\
0 & 0 \\
0 & 0
\end{tabular} & $\begin{array}{ll} & 0 \\
0 & \widehat{\delta} \\
0 & 0 \\
+1 & 0 \\
0 & 0 \\
0 & 0 \\
0\end{array}$ & $\begin{array}{l}\overrightarrow{0} \widehat{0} \\
0 \\
0 \\
+1 \\
0 \\
0 \\
0 \\
0\end{array}$ & 蒙 \\
\hline 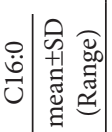 & 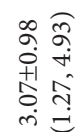 & 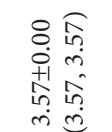 & 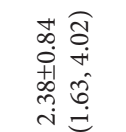 & 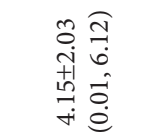 & 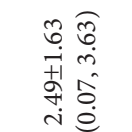 & 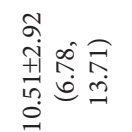 & 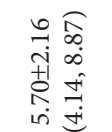 & 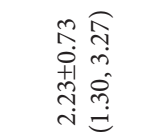 & 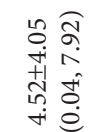 & $\ddot{\circ}$ \\
\hline 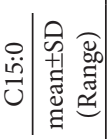 & 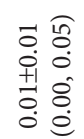 & $\begin{array}{ll} & \widehat{a} \\
0 & 0 \\
0 & 0 \\
+1 & 0 \\
0 & 0 \\
0 & 0\end{array}$ & $\begin{array}{l}8 \\
0 \\
0 \\
0 \\
0 \\
0 \\
0 \\
0 \\
0\end{array}$ & $\begin{array}{ll} & \widehat{0} \\
0 & 0 \\
0 & 0 \\
+1 & 0 \\
0 & 0 \\
0 & 0\end{array}$ & $\begin{array}{l}8 \\
8 \\
0 \\
0 \\
0 \\
0 \\
0 \\
0 \\
0\end{array}$ & 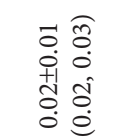 & 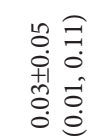 & $\begin{array}{ll} & \widehat{0} \\
0 & 0 \\
0 & 0 \\
0 & 0 \\
0 & 0 \\
0 & 0\end{array}$ & $\begin{array}{l}0 \\
0 \\
0 \\
0 \\
01 \\
0 \\
0 \\
0 \\
0\end{array}$ & $\vec{\circ}$ \\
\hline 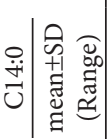 & 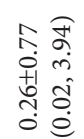 & 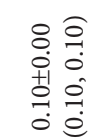 & 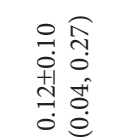 & 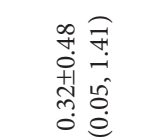 & 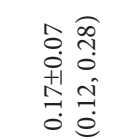 & 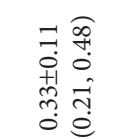 & 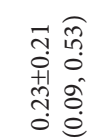 & 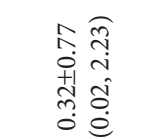 & 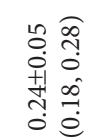 & $\stackrel{m}{0}$ \\
\hline 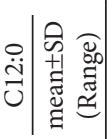 & 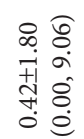 & $\begin{array}{l}8 \text { 合 } \\
0 \\
\text { +1 } \\
0 \\
0 \\
0 \\
0\end{array}$ & 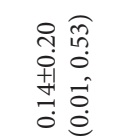 & 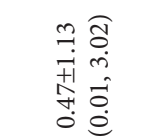 & 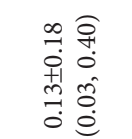 & $\begin{array}{l}n \\
0 \\
0 \\
0 \\
0 \\
+1 \\
0 \\
0 \\
0 \\
0\end{array}$ & 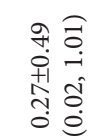 & 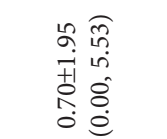 & 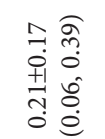 & $\stackrel{n}{\stackrel{0}{0}}$ \\
\hline 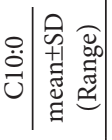 & $\begin{array}{ll}7 & \widehat{0} \\
0 & 0 \\
0 & 0 \\
+10 & 0 \\
0 & 0 \\
0 & 0\end{array}$ & $\begin{array}{l}8 \\
8 \\
0 \\
0 \\
+1 \\
0 \\
0 \\
0 \\
0\end{array}$ & 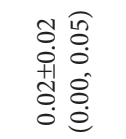 & $\begin{array}{ll} & 0 \\
0 & 0 \\
0 & 0 \\
+1 & 0 \\
0 & 0 \\
0 & 0 \\
0 & 0\end{array}$ & 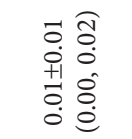 & 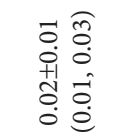 & $\begin{array}{ll} & \widehat{I} \\
0 & 0 \\
0 & 0 \\
+1 & 0 \\
0 & 0 \\
0 & 0\end{array}$ & 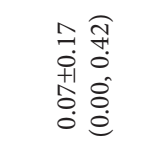 & 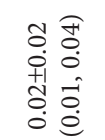 & 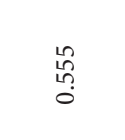 \\
\hline 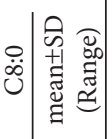 & 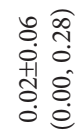 & 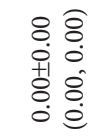 & $\begin{array}{l}\text { ro } \\
0 \\
0 \\
+0 \\
+1 \\
0 \\
0 \\
0 \\
0\end{array}$ & $\begin{array}{l}0.00 \\
0.0 \\
0 \\
0 \\
0 \\
0 \\
0 \\
0 \\
0 \\
0\end{array}$ & $\begin{array}{l}8 \hat{\sigma} \\
0 \\
0 \\
+1 \\
0 \\
0 \\
0 \\
0\end{array}$ & $\begin{array}{l}8 \hat{0} \\
0.0 \\
\text { +1 } \\
0 \\
0 \\
0 \\
0\end{array}$ & $\begin{array}{l}0 \widehat{0} \\
0 \\
0 \\
+1 \\
0 \\
0 \\
0 \\
0\end{array}$ & 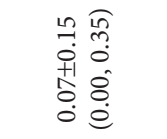 & 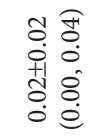 & în \\
\hline 4 & $\stackrel{\sim}{\sim}$ & $\sim$ & $\wedge$ & $\wedge$ & $H$ & H & H & $\infty$ & $m$ & \\
\hline $\begin{array}{c}\text { słənpold } \\
\text { [әә.д] }\end{array}$ & әуеЈ & גәҮрехว & әчวп[оу & 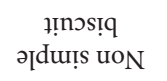 & !YЧse.I!d & $\begin{array}{l}\text { słonpo.dd } \\
\text { pəyn }\end{array}$ & 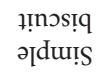 & әуез ə৪̊uоds & гәуем & 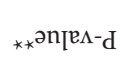 \\
\hline 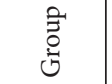 & & & & & [e!!.,snpuI & & & & & \\
\hline
\end{tabular}


Sugar, fat, saturated and trans fatty acid contents in cereal-based

\begin{tabular}{|c|c|c|c|c|c|c|c|}
\hline 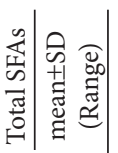 & 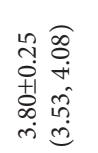 & 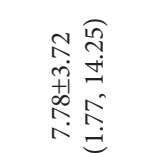 & $\begin{array}{l}0 \widehat{O} \\
\infty \\
\text { i } \\
+1 \\
\text { †f } \\
\text { in } \\
\text { in }\end{array}$ & 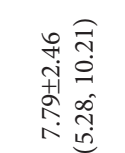 & 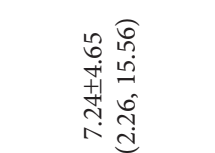 & 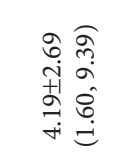 & 苋 \\
\hline 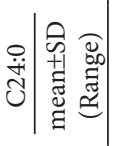 & $\begin{array}{lll} & & 0 \\
0 & 0 \\
0 & 0 \\
0 & 0 \\
10 & 0 \\
0 & 0 \\
0 & 0\end{array}$ & 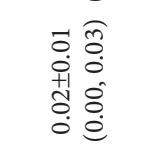 & 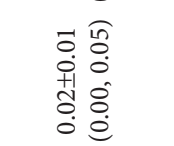 & 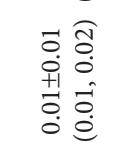 & 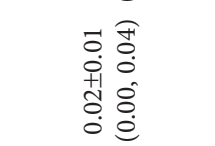 & 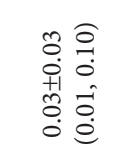 & 苞 \\
\hline 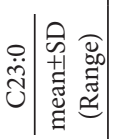 & $\begin{array}{ll}m & 0 \\
0 & 0 \\
0 & 0 \\
0 & 0 \\
+1 & 0 \\
0 & 0 \\
0 & 0 \\
0 & 0\end{array}$ & $\begin{array}{l}0 \\
0 \\
0 \\
0 \\
0 \\
0 \\
0 \\
0 \\
0\end{array}$ & 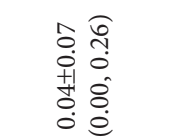 & 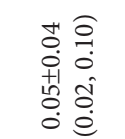 & 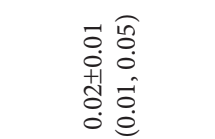 & 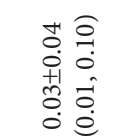 & $\stackrel{0}{9}$ \\
\hline 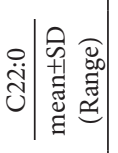 & $\begin{array}{ll} & \\
0 & \widehat{m} \\
0 & 0 \\
0 & 0 \\
+1 & 0 \\
0 & 0 \\
0 & 0 \\
0\end{array}$ & 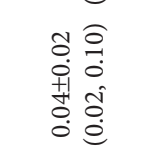 & $\begin{array}{l}m \text { I } \\
0 \\
0 \\
0 \\
+1 \\
0 \\
0 \\
0\end{array}$ & 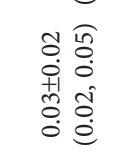 & $\begin{array}{l}m \\
0 \\
0 \\
0 \\
+1 \\
0 \\
0 \\
0 \\
0\end{array}$ & 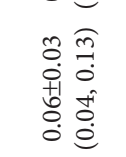 & oे \\
\hline 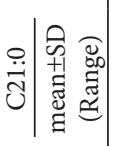 & 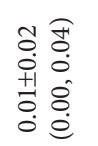 & 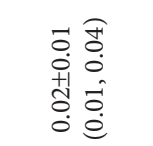 & $\begin{array}{ll} & 0 \\
0 & 0 \\
0 & 0 \\
+1 & 0 \\
0 & 0 \\
0 & 0\end{array}$ & $\begin{array}{l}\overrightarrow{0} \widehat{\delta} \\
0 \\
0 \\
0 \\
0 \\
0 \\
0 \\
0\end{array}$ & $\begin{array}{ll} & 0 \\
\delta & 0 \\
0 & 0 \\
+1 & 0 \\
0 & 0 \\
0 & 0 \\
0 & 0\end{array}$ & 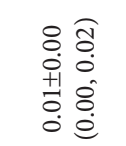 & $\frac{9}{0}$ \\
\hline 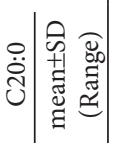 & $\begin{array}{ll}8 & 0 \\
0 & 0 \\
0 & 0 \\
11 & 0 \\
0 & 0 \\
0 & 0\end{array}$ & $\begin{array}{l}\tilde{0} \\
0 \\
0 \\
+1 \\
0 \\
0 \\
0\end{array}$ & $\begin{array}{ll} & \\
0 & \hat{a} \\
0 & 0 \\
0 & 0 \\
1 & 0 \\
0 & 0 \\
0 & 0\end{array}$ & 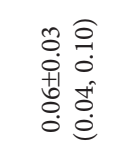 & 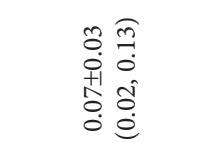 & 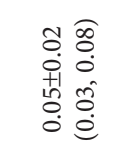 & $\stackrel{n}{\hat{n}}$ \\
\hline 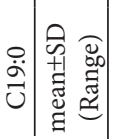 & $\begin{array}{ll}8 & \widehat{8} \\
0 & 0 \\
0 & 0 \\
0 & 0 \\
0 & 8 \\
0 & 0\end{array}$ & $\begin{array}{l}8 \\
8 \\
0 \\
0 \\
0 \\
0 \\
0 \\
0 \\
0\end{array}$ & $\begin{array}{l}8 \\
8 \\
0 \\
0 \\
0 \\
0 \\
0 \\
0\end{array}$ & $\begin{array}{l}8 \\
8 \\
0 \\
0 \\
0 \\
0 \\
0 \\
0 \\
0\end{array}$ & $\begin{array}{l}8 \\
8 \\
0 \\
0 \\
0 \\
0 \\
0 \\
0 \\
0\end{array}$ & $\begin{array}{l}8 \\
8 \\
0 \\
0 \\
0 \\
0 \\
0 \\
0\end{array}$ & 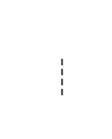 \\
\hline 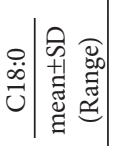 & 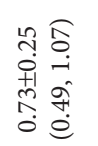 & 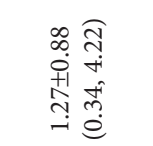 & 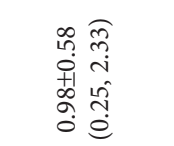 & 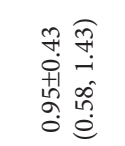 & 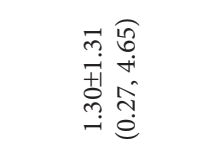 & 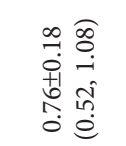 & ָे \\
\hline 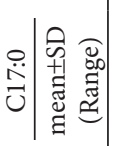 & $\begin{array}{ll} & \\
0 & \widehat{0} \\
0 & 0 \\
0 & 0 \\
0 & 0 \\
0 & 0 \\
0 & 0 \\
0 & 0\end{array}$ & 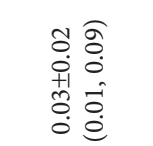 & $\begin{array}{ll} & \\
0 & \hat{o} \\
0 & 0 \\
0 & 0 \\
0 & 0 \\
0 & 0 \\
0 & 0 \\
0\end{array}$ & 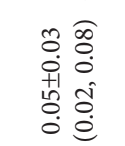 & 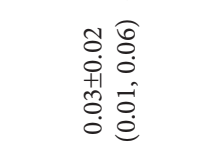 & 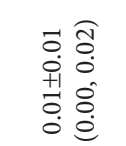 & 范 \\
\hline 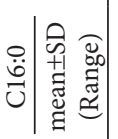 & 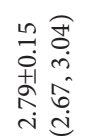 & 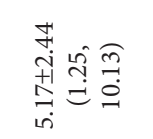 & 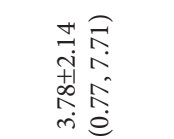 & 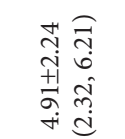 & 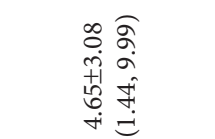 & 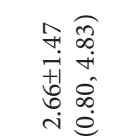 & ò \\
\hline 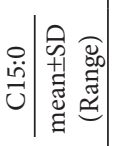 & $\begin{array}{ll} & \\
0 & \widehat{\delta} \\
0 & 0 \\
0 & 0 \\
0 & 0 \\
0 & 0 \\
0 & 0\end{array}$ & $\begin{array}{l}m \\
0 \\
0 \\
0 \\
+1 \\
0 \\
0 \\
0 \\
0\end{array}$ & 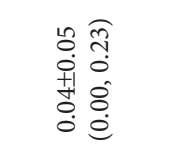 & 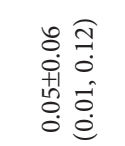 & $\begin{array}{l}+\sqrt{1} \\
+0 \\
0 \\
+1 \\
0 \\
0 \\
0 \\
0\end{array}$ & 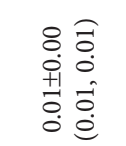 & ্ָড \\
\hline 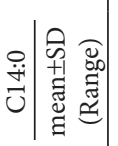 & $\begin{array}{ll} & 0 \\
0 & 0 \\
0 & 0 \\
+1 & 0 \\
\text { I } & 8 \\
0 & 0\end{array}$ & 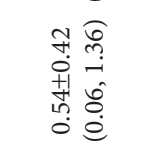 & 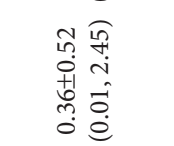 & 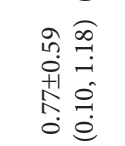 & 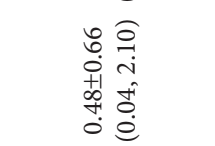 & 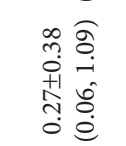 & $\hat{o}_{0}^{\infty}$ \\
\hline 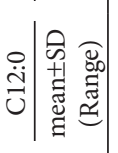 & $\begin{array}{ll} & \\
0 & 0 \\
0 & 0 \\
0 & 0 \\
+1 & \hat{s} \\
0 & 0 \\
0 & 0\end{array}$ & 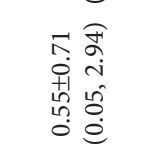 & $\begin{array}{ll} & \\
0 & 0 \\
0 & \infty \\
0 & 0 \\
+1 & 0 \\
0 & 0 \\
0 & 0 \\
0 & 0\end{array}$ & 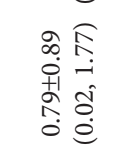 & 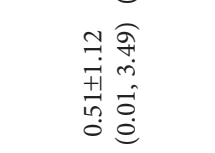 & 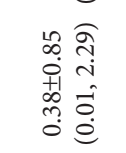 & $\stackrel{o}{o}$ \\
\hline 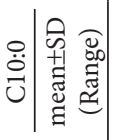 & $\begin{array}{l}\vec{a} \\
0 \\
0 \\
0 \\
+1 \\
0 \\
0 \\
0 \\
0\end{array}$ & $\begin{array}{l}n \\
0 \\
0 \\
0 \\
+1 \\
11 \\
0 \\
0 \\
0 \\
0\end{array}$ & 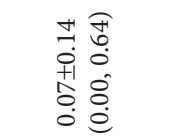 & 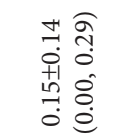 & 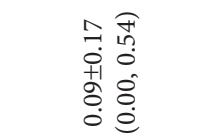 & 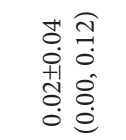 & $\stackrel{2}{0}$ \\
\hline 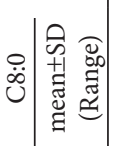 & $\begin{array}{l}8 \\
0 \\
0 \\
0 \\
+1 \\
0 \\
0 \\
0 \\
0\end{array}$ & $\begin{array}{l}+0 \\
0:= \\
00 \\
+10 \\
00 \\
\dot{0} 0\end{array}$ & 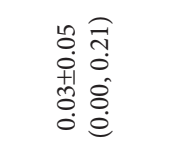 & $\begin{array}{l}\text { no } \\
00 \\
0 \\
+10 \\
0 \\
0 \\
0 \\
0\end{array}$ & 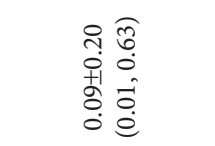 & \begin{tabular}{ll} 
& \multicolumn{1}{c}{} \\
0 & \\
0 & 0 \\
0 & 0 \\
01 & 0 \\
0 & 0 \\
0 & 0
\end{tabular} & 产 \\
\hline z & in & $\vec{\sim}$ & $\approx$ & m & $a$ & $n$ & \\
\hline $\begin{array}{c}\text { słэnpo.d } \\
\text { [एә.əว }\end{array}$ & әуеว & 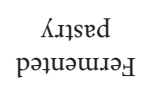 & K.nsed paun & әчәп[оу & 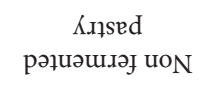 & 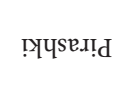 & әп \\
\hline 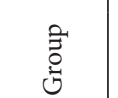 & & & & 鬲 & & & \\
\hline
\end{tabular}




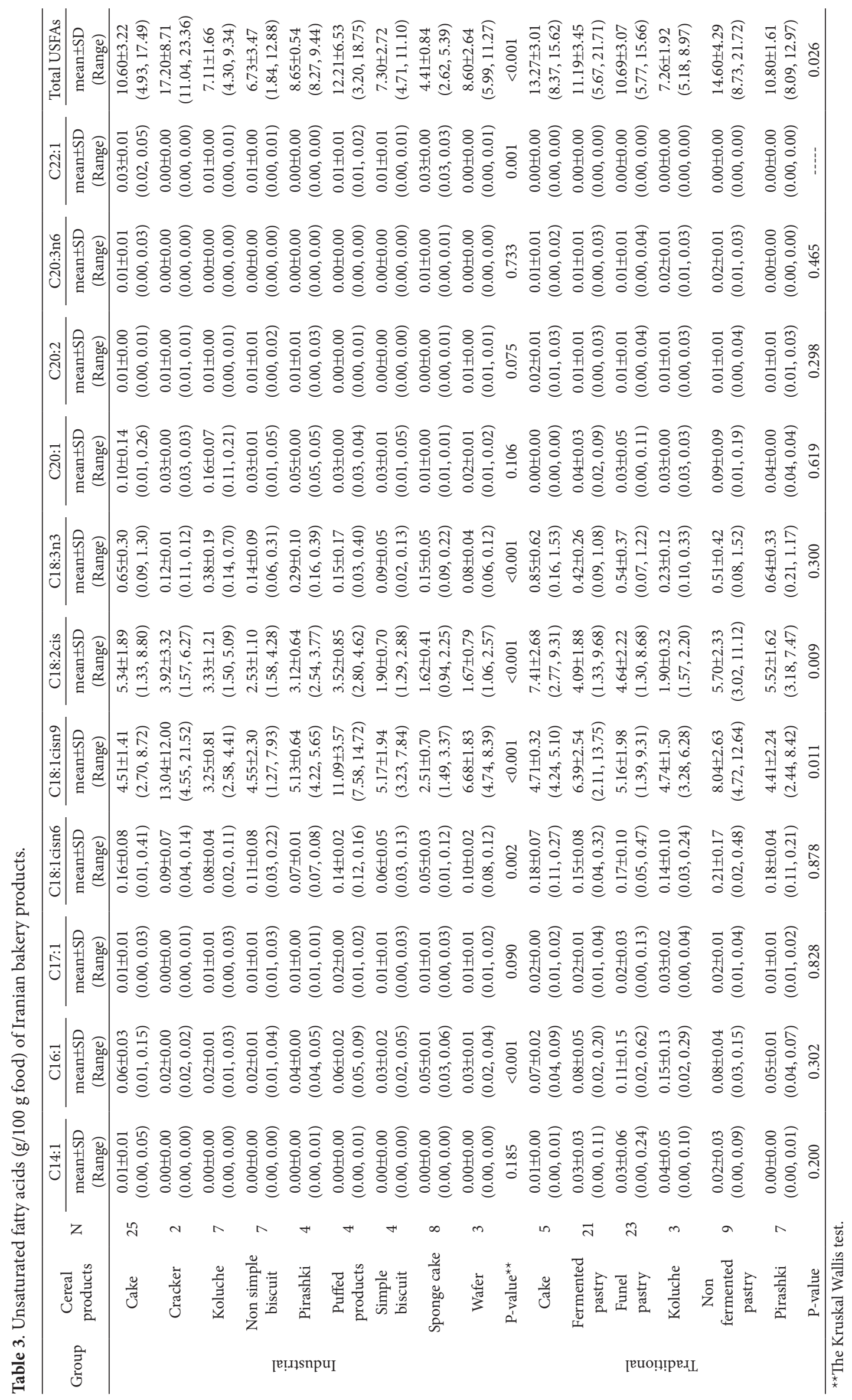


Table 4. Total fat and sugar ( $\mathrm{g} / 100 \mathrm{~g}$ food) of Iranian bakery products.

\begin{tabular}{|c|c|c|c|c|c|c|}
\hline \multirow{2}{*}{ Group } & \multirow{2}{*}{ Cereal products } & \multirow{2}{*}{$\mathrm{N}$} & Total fat & \multirow{2}{*}{ Target limit $\dagger$} & Total sugar & \multirow{2}{*}{ Target limit } \\
\hline & & & Mean \pm SD (Range) & & Mean \pm SD (Range) & \\
\hline \multirow{9}{*}{ Industrial } & Cake & 25 & $15.83 \pm 5.56(9.25,46.10)$ & $\operatorname{Min}=13$ & $25.15 \pm 4.48(18.48,34.12)$ & $\operatorname{Min}=25$ \\
\hline & Cracker & 2 & $19.59 \pm 5.77(18.61,27.89)$ & $\operatorname{Min}=10$ & $20.66 \pm 17.47(8.31,33.01)$ & $\operatorname{Max}=8$ \\
\hline & Koluche & 7 & $10.94 \pm 1.71(12.42,39.38)$ & $\operatorname{Min}=16$ & $27.31 \pm 5.07(20.74,34.79)$ & $\operatorname{Min}=32$ \\
\hline & Non simple biscuit & 7 & $12.83 \pm 4.86(12.11,23.18)$ & $\operatorname{Min}=11$ & $20.72 \pm 3.28(14.18,24.70)$ & $\operatorname{Min}=18$ \\
\hline & Pirashki & 4 & $12.76 \pm 0.34(19.10,31.81)$ & $*$ & $17.17 \pm 9.38(9.92,30.95)$ & * \\
\hline & Puffed products & 4 & $24.92 \pm 7.86(15.84,23.11)$ & $\operatorname{Min}=15$ & $00 \pm 0.0(0.00,0.00)$ & * \\
\hline & Simple biscuit & 4 & $15.72 \pm 5.23(12.90,18.74)$ & $\operatorname{Min}=17$ & $18.28 \pm 3.45(16.09,23.36)$ & $\operatorname{Min}=18$ \\
\hline & Sponge cake & 8 & $8.99 \pm 2.95(0.09,22.41)$ & * & $29.92 \pm 6.02(18.89,36.88)$ & $\operatorname{Min}=27$ \\
\hline & Wafer & 3 & $16.61 \pm 3.25(23.02,29.00)$ & * & $21.48 \pm 1.06(20.71,22.69)$ & * \\
\hline \multirow{8}{*}{ Traditional } & P-value ${ }^{* *}$ & & 0.020 & & 0.011 & \\
\hline & Cake & 5 & $17.44 \pm 3.07(15.40,31.86)$ & $\operatorname{Min}=13$ & $24.20 \pm 3.56(20.55,28.83)$ & $\operatorname{Min}=25$ \\
\hline & Fermented pastry & 21 & $19.41 \pm 6.30(8.92,43.33)$ & $\operatorname{Min}=20$ & $23.12 \pm 8.50(12.55,44.00)$ & * \\
\hline & Funnel pastry & 23 & $16.74 \pm 4.56(1.92,44.90)$ & $\operatorname{Min}=18$ & $30.38 \pm 13.11(7.92,51.70)$ & $\operatorname{Min}=20$ \\
\hline & Koluche & 3 & $15.30 \pm 3.61(26.50,28.47)$ & $\operatorname{Min}=16$ & $25.07 \pm 5.08(20.07,30.23)$ & $\operatorname{Min}=32$ \\
\hline & Non fermented pastry & 9 & $22.24 \pm 7.02(17.77,35.24)$ & $\operatorname{Min}=25$ & $28.19 \pm 13.22(11.78,50.13)$ & * \\
\hline & Pirashki & 7 & $15.38 \pm 3.27(20.50,29.13)$ & * & $11.12 \pm 1.89(9.07,14.49)$ & * \\
\hline & $\mathrm{P}$-value & & 0.736 & & 0.022 & \\
\hline
\end{tabular}

$\dagger$ The permitted limit of fat and sugar contents ( $\mathrm{g} / 100 \mathrm{~g}$ ) set by the Institute of Standards and Industrial Research of Iran (ISIRI); ${ }^{*}$ No limits determined by the (ISIRI); ${ }^{* *}$ The Kruskal Wallis test.

with the permitted limit set by the Iranian National Standards Organization, the mean levels of total fat found in industrial category (64 samples) such as simple biscuit, pancake, koluche, fermented pastry, wafer, and puffed product were in accordance with those permitted levels, whereas $80 \%, 50 \%$ and $80 \%$ of the non-simple biscuit, cracker, and cake, complied with those of permitted levels set by Institute of Standards and Industrial Research of Iran, respectively $(2553 ; 2006,14728 ; 2012,2554$; $2018,2880 ; 2016,37 ; 2016,592 ; 2020,3682 ; 2018)$. Overall, the traditional bakery cereal products (68 samples) met the total fat target level of Iranian National Standards Organization (3493).

Total sugar ranged from $11.12 \pm 1.89 \%$ to $30.38 \pm 13.11 \%$ in fifteen bakery products. The total sugar content of the traditional funnel $(30.38 \pm 13.11 \%)$ and non-fermented pastry $(28.19 \pm 13.22 \%)$ was significantly higher than those of other products, but they compliant with those of permitted levels set by the Iranian National Standards Organization. Nevertheless, the total sugar content of industrial samples was $100 \%$ compliant with the permitted level Iranian National Standards Organization. Iranian National Standards Organization has not set target limits for pirashki, sponge cake, and wafer. Comparison of total sugar and fat in industrial and traditional bakery products showed that except pirashki sample, no differences were observed between those mean levels ( $\mathrm{p} \geq 0.05)$.

Likewise, Cercaci et al. (2006) reported that total fat content in European bakery products ranged from 8.9 to 39.7 (g/100 g product) (Cercaci et al., 2006). Chung et al. (2013) evaluated the mean levels of total lipids in Hong Kong bakery products and reported a range of 6.4-29 (g/100 g product) (Chung et al., 2013). According to FAO, a minimum of $15 \%$ of adults' total energy should be provided from fat intake that is adequate to gain fatsoluble vitamins and essential fatty acids (Fats)fats). There are many regions that solely a maximum of $5 \%-10 \%$ of calories/ day is provided by sugar. The UK government has suggested reducing the total sugar content by $20 \%$ in bakery products by 2020 (Sahin et al., 2019). Excessive sugar consumption is correlated with obesity, insulin resistance, type 2 diabetes, fatty liver, and dental caries (O'Sullivan, 2020; World Health Organization, 2003). Besides, as stated before, high-fat intakes are also associated with N.C.D.s, including coronary heart disease (CHDs), high blood cholesterol, and obesity. Some other diseases, such as high blood pressure, unusual blood fat, high blood clotting, increase blood insulin, and diabetes (type 2 ), also occur following obesity. Considering that $82 \%$ of Iran's annual deaths are connected to NCDs, reducing fat and sugar consumption has always been suggested (Hadian et al., 2020; World Health Organization, 2003). One should note that this suggestion does not apply solely to consumers and the food industry to control and reduce fat and sugar in the formulation of bakery products (Barclay et al., 2008).

\subsection{Total fat and sugar intake}

A national survey on food consumption (2002) has demonstrated that the mean per capita intake of cake, crackers, biscuits, and traditional Iranian confectionaries for the Iranian communities was $8 \mathrm{~g} /$ day. The total fat contents in industrial bakery products were significantly different and ranged from $46.10 \%$ (cake) to $0.09 \%$ (sponge cake) $(\mathrm{P}=0.02)$. Similarly, a comparison of total sugar contents between this group was statistically significant $(\mathrm{P}=0.011)$. The range of total fat and sugar contents in traditional samples were between $1.92 \%$ to $44.90 \%$ and $7.92 \%$ to $51.70 \%$, respectively. When the contents of total fat between the traditional group were compared, the differences were not significant $(\mathrm{P}=0.736)$.

The present study indicated that total bakery products' mean total fat and sugar contents were $23.1 \pm 8.5 \%$ and $24.72 \pm$ 
$9.4 \%$, with the maximum levels $46.10 \%$ and $51.70 \%$, respectively. Based on this mean consumption per capita of traditional bakery products in Iran $(8 \mathrm{~g})$, the means of total fat and sugar intake from these samples were estimated to be 1.85 and $1.98 \mathrm{~g} /$ day, respectively. Therefore the maximum daily intake of total fat and sugar per capita from cereal-based bakery products is estimated at 3.69 and $4.14 \mathrm{~g}$ per person, respectively. In a case-control study in Iran, Yari et al. (2020) revealed that there might be a relationship between energy-dense nutrient-poor snacks and metabolic abnormalities risk such as non-alcoholic fatty liver disease (Yari et al., 2020).

\section{Conclusion}

In the present study, the results indicated that the level of fat, saturated, and trans fatty acids of bakery products in Iran were not higher than those reported in other countries' bakery products. Nevertheless, overconsumption of cereal-based baked products because of fat and sugar is linked with non-communicable diseases. For this reason, accurate health intervention plans and strategies should be developed by authorized organizations not only to the consumer but also needs the food industry and confectioners to reduce sugar and fat in product formulation. Regarding Iranian National Standards Organization legislation that addresses minimum limits set of fat and sugar levels for some Iranian cereal-based- bakery products, it is strongly recommended that the minimum permitted target levels for sugar and fat should be modified and replaced with the maximum limits by the Iranian National Standards Organization by considering public health.

\section{Acknowledgements}

This study was supported by the National Nutrition and Food Technology Research Institute, Shahid Beheshti University of Medical Sciences, Iran. Grant No. 1448 and 1288.

\section{References}

Albuquerque, T. G., Santos, J., Silva, M. A., Oliveira, M. B. P., \& Costa, H. S. (2018). An update on processed foods: Relationship between salt, saturated and trans fatty acids contents. Food Chemistry, 267, 75-82. http://dx.doi.org/10.1016/j.foodchem.2018.01.029. PMid:29934192.

Alioğlu, T., \& Özülkü, G. (2020). Evaluation of whole wheat flour sourdough as a promising ingredient in short dough biscuits. Food Science and Technology. In press, 1-8.

Amini, M., Dadkhah-Piraghaj, M., Abtahi, M., Abdollahi, M., Houshiarrad, A., \& Kimiagar, M. (2014). Nutritional assessment for primary school children in Tehran: An evaluation of dietary pattern with emphasis on snacks and meals consumption. International Journal of Preventive Medicine, 5(5), 611-616. PMid:24932393.

Arcand, J., Scourboutakos, M. J., Au, J. T., \& L’Abbe, M. R. (2014). Trans Fatty acids in the Canadian food supply: an updated analysis. The American Journal of Clinical Nutrition, 100(4), 1116-1123. http:// dx.doi.org/10.3945/ajcn.114.088732. PMid:25099549.

Asgary, S., Nazari, B., Sarrafzadegan, N., Parkhideh, S., Saberi, S., Esmaillzadeh, A., \& Azadbakht, L. (2009). Evaluation of fatty acid content of some Iranian fast foods with emphasis on trans fatty acids. Asia Pacific Journal of Clinical Nutrition, 18(2), 187-192. PMid:19713177.
Barclay, A. W., Petocz, P., McMillan-Price, J., Flood, V. M., Prvan, T., Mitchell, P., \& Brand-Miller, J. C. (2008). Glycemic index, glycemic load, and chronic disease risk-a meta-analysis of observational studies. The American Journal of Clinical Nutrition, 87(3), 627-637. http://dx.doi.org/10.1093/ajcn/87.3.627. PMid:18326601.

Belc, N., Smeu, I., Macri, A., Vallauri, D., \& Flynn, K. (2019). Reformulating foods to meet current scientific knowledge about salt, sugar and fats. Trends in Food Science \& Technology, 84, 25-28. http://dx.doi. org/10.1016/j.tifs.2018.11.002.

Cercaci, L., Conchillo, A., Rodriguez-Estrada, M. T., Ansorena, D., Astiasaran, I., \& Lercker, G. (2006). Preliminary study on health-related lipid components of bakery products. Journal of Food Protection, 69(6), 1393-1401. http://dx.doi.org/10.4315/0362-028X-69.6.1393. PMid:16786862.

Chajès, V., Thiébaut, A. C., Rotival, M., Gauthier, E., Maillard, V., Boutron-Ruault, M.-C., Joulin, V., Lenoir, G. M., \& Clavel-Chapelon, F. (2008). Association between serum trans-monounsaturated fatty acids and breast cancer risk in the E3N-EPIC study. American Journal of Epidemiology, 167(11), 1312-1320. http://dx.doi.org/10.1093/aje/ kwn069. PMid:18390841.

Chung, S. W., Tong, S., Lin, V. F., Chen, M. Y., Ma, J. K., Xiao, Y., \& Ho, Y. (2013). Trans fatty acids in the Hong Kong Food Supply. Journal of Chemistry, 2013, 2013. http://dx.doi.org/10.1155/2013/327582.

Colón-Ramos, U., Monge-Rojas, R., \& Campos, H. (2014). Impact of WHO recommendations to eliminate industrial trans-fatty acids from the food supply in Latin America and the Caribbean. Health Policy and Planning, 29(5), 529-541. http://dx.doi.org/10.1093/ heapol/czt034. PMid:24150503.

Costa, N., Cruz, R., Graça, P., Breda, J., \& Casal, S. (2016). Trans fatty acids in the Portuguese food market. Food Control, 64, 128-134. http://dx.doi.org/10.1016/j.foodcont.2015.12.010. PMid:27274619.

Dordevic, D., Rosa, M. C. C., Javurkova, Z., Buchtova, H., \& Jancikova, S. (2020). Consumers' response to different shelf life food labelling. Quality Assurance and Safety of Crops \& Foods, 12(3), 24-34. http:// dx.doi.org/10.15586/QAS2019.646.

Folch, J., Lees, M., \& Stanley, G. S. (1957). A simple method for the isolation and purification of total lipides from animal tissues. The Journal of Biological Chemistry, 226(1), 497-509. http://dx.doi. org/10.1016/S0021-9258(18)64849-5. PMid:13428781.

Fuentes-Arismendy, F., Rodriguez-Sandoval, E., Mejia-Villota, A., Velez-Uribe, T., \& Hernandez, V. (2021). Development of baked snack with fats and proteins powder mixtures as a fresh cheese substitute. Food Science and Technology. In press.

Hadian, Z., Feizollahi, E., Khosravi, K., Mofid, V., \& Rasekhi, H. (2020). Salt intake from traditional breads: a public health challenge for decreasing non-communicable diseases in Iran. Current Nutrition and Food Science, 16(8), 1278-1284. http://dx.doi.org/10.2174/157 3401316666200309150947

Hercegová, D., Ivanišová, E., Zagula, G., Terentjeva, M., Kročko, M., Tvrdá, E., \& Kačániová, M. (2019). Technological, phytochemical and sensory profile of honey biscuits made from buckwheat, rye, spelt and wheat flour. Quality Assurance and Safety of Crops \& Foods, 11(4), 333-340. http://dx.doi.org/10.3920/QAS2018.1376.

Hooper, L., Abdelhamid, A., Moore, H. J., Douthwaite, W., Skeaff, C. M., \& Summerbell, C. D. (2012). Effect of reducing total fat intake on body weight: systematic review and meta-analysis of randomised controlled trials and cohort studies. BMJ (Clinical Research Ed.), 345(dec06 1), 345. http://dx.doi.org/10.1136/bmj. e7666. PMid:23220130. 
Institute of Standards and Industrial Research of Iran - ISIRI. (2017). 3493 Cookies and Pastry Specifications and Test Methods. 2nd. Karaj, Tehran: ISIRI.

Institute of Standards and Industrial Research of Iran. (2016). 2553 Cake - Specification and Test Methods. ICS: 67.060. 3th ed..

Institute of Standards and Industrial Research of Iran. Cake - Specification and test methods (2553; 2006); Pancake - Specifications and Test Methods (14728; 2012); Koluche-Specifications and test methods (2554; 2018); Doughnut-Specifications and test methods (16980; 2013); Puffed productsbased on cereal grit and flour-Specificationsand test methods (2880; 2016); Biscuit-Specifications and test methods (37; 2016); Wafer-Specificationsand test methods-Amd.No.2 (592; 2020); Cracker - Specification and test methods (3682; 2018).

International Organization for Standardization - ISO. (2009). ISO 712:2009: Cereals and Cereals Products- Determination of Moisture Content-reference Method. ISO.

International Organization for Standardization - ISO. (2014). ISO 129661:2014. Animal and vegetable fats and oils-Gas chromatography offatty acid methyl esters-Part 1: Guidelines on modern gas chromatography of fatty acid methyl esters. ISO.

Karabulut, I. (2007). Fatty acid composition of frequently consumed foods in Turkey with special emphasis on trans fatty acids. International Journal of Food Sciences and Nutrition, 58(8), 619-628. http://dx.doi. org/10.1080/09637480701368967. PMid:17852509.

Lucas, B. F., Rosa, A. P. C., Carvalho, L. F., Morais, M. G., Santos, T. D., \& Costa, J. A. V. (2020). Snack bars enriched with Spirulina for schoolchildren nutrition. Food Science and Technology (Campinas), 40(Suppl. 1), 146-152. http://dx.doi.org/10.1590/fst.06719.

Menaa, F., Menaa, A., Tréton, J., \& Menaa, B. (2013). Technological approaches to minimize industrial trans fatty acids in foods. Journal of Food Science, 78(3), R377-R386. http://dx.doi.org/10.1111/17503841.12055. PMid:23458752.

Mossoba, M., Kramer, J., Delmonte, P., Yurawecz, M., \& Rader, J. (2003). AOAC Official Method 996.06, fat (total, saturated, and unsaturated in foods), hydrolytic extraction gas chromatographic method, first action 1996, revised 2001. Urbana, IL: AOCS Press.

Mozaffarian, D., Abdollahi, M., Campos, H., Houshiarrad, A., \& Willett, W. (2007). Consumption of trans fats and estimated effects on coronary heart disease in Iran. European Journal of Clinical Nutrition, 61(8), 1004-1010. http://dx.doi.org/10.1038/sj.ejcn.1602608. PMid:17268422.

Nazari, B., Asgary, S., \& Azadbakht, L. (2012). Fatty acid analysis of Iranian junk food, dairy, and bakery products: Special attention to trans-fats. Journal of Research in Medical Sciences: The Official Journal of Isfahan University of Medical Sciences, 17(10), 952.

Nishida, C., Uauy, R., Kumanyika, S., \& Shetty, P. (2004). The joint WHO/FAO expert consultation on diet, nutrition and the prevention of chronic diseases: process, product and policy implications. Public Health Nutrition, 7(1a), 245-250. http://dx.doi.org/10.1079/ PHN2003592. PMid:14972063.

O’Fallon, J. V., Busboom, J., Nelson, M., \& Gaskins, C. (2007). A direct method for fatty acid methyl ester synthesis: application to wet meat tissues, oils, and feedstuffs. Journal of Animal Science, 85(6), 1511-1521. http://dx.doi.org/10.2527/jas.2006-491. PMid:17296772.

O'Sullivan, M. (2020). Salt, Fat and Sugar Reduction: Sensory Approaches for Nutritional Reformulation of Foods and Beverages. United Kingdom: Woodhead Publishing.

Omeroglu, P. Y., \& Ozdal, T. (2020). Fatty acid composition of sweet bakery goods and chocolate products and evaluation of overall nutritional quality in relation to the food label information. Journal of Food Composition and Analysis, 88, 103438. http://dx.doi. org/10.1016/j.jfca.2020.103438.

Pinto, V. R. A., Araújo, L. G., Soares, L. S., Dantas, M. I. S., Lucia, S. M. D., Souza, T. D., Melo, L. F., Minim, V. P. R., \& Bressan, J. (2019). Preference mapping to assess the effect of information on the acceptability of snack bars. Food Science and Technology (Campinas), 39(Suppl. 1), 316-323. http://dx.doi.org/10.1590/fst.03418.

Priego-Capote, F., Ruiz-Jiménez, J., \& De Castro, M. L. (2007). Identification and quantification of trans fatty acids in bakery products by gas chromatography-mass spectrometry after focused microwave Soxhlet extraction. Food Chemistry, 100(2), 859-867. http://dx.doi.org/10.1016/j.foodchem.2005.10.001.

Pușcaș, A., Mureșan, V., Socaciu, C., \& Muste, S. (2020). Oleogels in Food: A review of current and potential applications. Foods, 9(1), 70. http://dx.doi.org/10.3390/foods9010070. PMid:31936353.

Richter, E. K., Shawish, K. A., Scheeder, M. R., \& Colombani, P. C. (2009). Trans fatty acid content of selected Swiss foods: the TransSwissPilot study. Journal of Food Composition and Analysis, 22(5), 479-484. http://dx.doi.org/10.1016/j.jfca.2009.01.007.

Saadeh, C., Toufeili, I., Habbal, M. Z., \& Nasreddine, L. (2015). Fatty acid composition including trans-fatty acids in selected cerealbased baked snacks from Lebanon. Journal of Food Composition and Analysis, 41, 81-85. http://dx.doi.org/10.1016/j.jfca.2015.01.014.

Saghafi, Z., Zargaraan, A., Tabibiazar, M., \& Hosseini, H. (2018). Is trans fatty acid still an issue for policy makers in Iran? A Technical Report. Nutrition and Food Sciences Research, 5(2), 47-51. http:// dx.doi.org/10.29252/nfsr.5.2.47.

Sahin, A. W., Zannini, E., Coffey, A., \& Arendt, E. K. (2019). Sugar reduction in bakery products: Current strategies and sourdough technology as a potential novel approach. Food Research International, 126, 108583. http://dx.doi.org/10.1016/j.foodres.2019.108583. PMid:31732069.

Sarifudin, A., Afifah, N., Indrianti, N., Desnilasari, D., Kristanti, D., Ratnawati, L., \& Ekafitri, R. (2021). Optimization of banana bar formulation to provide a nourishing snack for toddlers using response surface methodology. Food Science and Technology (Campinas), 41(Suppl. 1), 21-28. http://dx.doi.org/10.1590/fst.07620.

Slattery, M. L., Benson, J., Ma, K.-N., Schaffer, D., \& Potter, J. D. (2001). Trans-fatty acids and colon cancer. Nutrition and Cancer, 39(2), 170175. http://dx.doi.org/10.1207/S15327914nc392_2. PMid:11759276.

Stanner, S., \& Spiro, A. (2020). Public health rationale for reducing sugar: Strategies and challenges. Nutrition Bulletin, 45(3), 253-270. http://dx.doi.org/10.1111/nbu.12460.

Trattner, S., Becker, W., Wretling, S., Öhrvik, V., \& Mattisson, I. (2015). Fatty acid composition of Swedish bakery products, with emphasis on trans-fatty acids. Food Chemistry, 175, 423-430. http://dx.doi. org/10.1016/j.foodchem.2014.11.145. PMid:25577101.

Vicario, I. M., Griguol, V., \& León-Camacho, M. (2003). Multivariate characterization of the fatty acid profile of Spanish cookies and bakery products. Journal of Agricultural and Food Chemistry, 51(1), 134-139. http://dx.doi.org/10.1021/jf0258297. PMid:12502397.

World Health Organization - WHO. (2003). Diet, nutrition, and the prevention of chronic diseases: report of a joint WHO/FAO expert consultation (Vol. 916). Geneva: World Health Organization.

World Health Organization - WHO. (2003). Diet, nutrition, and the prevention of chronic diseases: report of a joint WHO/FAO expert consultation (Vol. 916). Geneva: World Health Organization.

World Health Organization - WHO. (2015). Guideline: sugars intake for adults and children. Geneva: World Health Organization. 
Yari, Z., Cheraghpour, M., Aghamohammadi, V., Alipour, M., Ghanei, N., \& Hekmatdoost, A. (2020). Energy-dense nutrient-poor snacks and risk of non-alcoholic fattyliver disease: a case-control study in Iran. BMC Research Notes, 13, 1-5.
Zuchowska-Grzywacz, M., \& Kowalska, M. (2019). Trans fatty acids in food-current legal regulations as protections for consumers and food manufacturers. Acta Alimentaria, 48(1), 105-114. http://dx.doi. org/10.1556/066.2019.48.1.12. 